\title{
Gut ischaemia
}

\author{
U Haglund
}

\begin{abstract}
Intestinal mucosal injury that results from local ischaemia can be detected by early increases in gut permeability, followed by later morphological, histological, and biochemical abnormalities. Local adaptive mechanisms (for example increased oxygen extraction) can cope with reductions in blood flow of up to $50 \%$, as may occur during episodes of septic shock or cardiac tamponade. Why then does hypoxic injury develop? The peculiar vascular anatomy of the villi allows for oxygen short circuiting to occur at their base, when blood flow is low. Although overall oxygen extraction efficiency may be high, regional hypoxia at the villus tip may, paradoxically occur. The severity of reperfusion injury depends on the duration of preceding hypoxia. Free radical generation through the hypoxanthine xanthine oxidase system is important in mediating cellular damage. In addition, luminal aggressive factors (for example, pancreatic proteases) may cause mucosal damage, as suggested by earlier studies. More recent studies in pigs suggests that pancreatic duct ligation merely delays, but does not prevent development of gut reperfusion injury. Enteral nutrition should benefit patients with the ischaemic intestine because in comparison with total parental nutrition, it stimulates regional blood flow, and attenuates mucosal injury. There are no randomised trials to verify this, but use of tonometry to monitor local ischaemia may help resolve the issue.
\end{abstract}

(Gut 1994; supplement 1: S73-S76)

The intact gut mucosa serves as a barrier between the non-sterile lumen and the sterile interior of the body. Micro-organisms may sometimes penetrate this barrier - a process referred to as translocation. ${ }^{1}$ According to present knowledge, translocation in the normal healthy subject causes only a limited bacteraemic/endotoxinaemic challenge, which can be cleared by the defence systems (mesenteric lymph nodes and the hepatic reticuloendothelial system). During pathological states, however, leakage of bacteria through the more permeable gut mucosa may be increased, resulting in a significant septic condition. After trauma, in critical illness or septic states, the intestinal mucosal barrier may be impaired or possibly broken down completely. As a consequence, the possibility for micro-organisms to invade the body is greatly enhanced. In addition, other toxic substances such as myocardial depressant substances and proteolytic enzymes might be released from the intestine. ${ }^{2}$ Mucosal ischaemia is believed to be a common denominator for the processes that cause enhanced mucosal barrier permeability. The participation of mucosal ischaemia in intestinal mucosal injury and the possible influence by enteral nutrition on these conditions are currently being studied.

\section{Ischaemic intestinal mucosal injury (Table)}

The first detectable sign of intestinal mucosal injury in ischaemia is increased capillary permeability. With longterm ischaemia, the mucosal permeability will be further increased with subsequent mucosal epithelial cell injury becoming morphologically detectable. It is important to consider the intestinal mucosal injury process as a continuum of injury ranging from increased permeability to tissue destruction (Fig 1). The extent and duration of ischaemia will determine the depth of the tissue injury ${ }^{3}$ (Fig 2).

Intestinal ischaemia as seen, for example, in the septic, critically ill patient probably does not cause damage to the gut beyond the superficial part of the mucosa. Intestinal strangulation, mesenteric vascular obstruction, and so called non-occlusive intestinal ischaemia might cause additional injury to the deeper layers of the intestinal wall. In the patient suffering with shock, mucosal injury is confined to the villi. The first morphological sign of tissue injury is the lifting of the epithelial cells at the very tip of the villi. ${ }^{4}$ With more severe or more prolonged ischaemia, or both, the epithelial cells along the sides of the villus are lifted until the epithelial

Mechanism of intestinal mucosal injury

Ischaemic injury

Decreased oxygen delivery

Reduced intestinal (mucosal) blood flow

Increased short circuiting of oxygen in the villus counter-

current exchanger

Increased needs of oxygen

Reperfusion injury

Normal mucosa

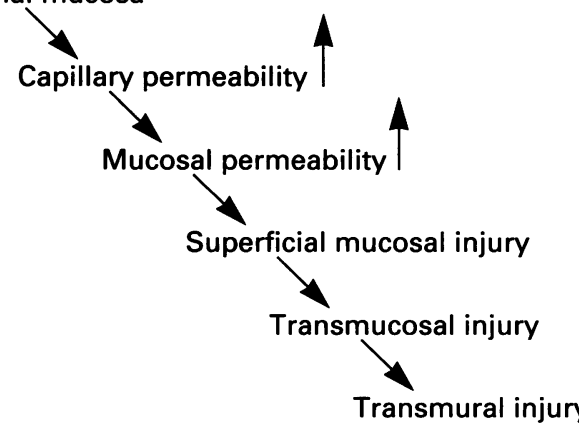

Figure 1: The continuum of intestinal ischaemic injury from normal mucosa, permeability changes to transmural infarction. 


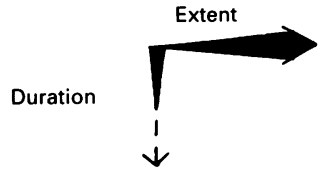

Figure 2: Intestinal ischaemia is characterised by its extent and its duration. cells are finally lost and the villus core disintegrates. Chiu et al describe an injury related grading system that has been widely used ${ }^{4}$ because their various grades have shown to be proportional to the extent and duration of the ischaemia. Even in situations where the villi are severely damaged, healing might occur quite rapidly if measures to provide adequate mucosal tissue oxygenation were instituted. Thus, Park et al ${ }^{5}$ showed that epithelial lifting returns to normal within six hours. Villi that had lost their epithelial cells were shown to be covered and to maintain a fairly normal appearance within 18 hours. $^{5}$

\section{Ischaemic mucosal injury}

During ischaemia, the intestinal mucosa may be injured as a result of several mechanisms acting alone or in concert. Ischaemia may cause intestinal injury, which may increase oxygen demands or decrease oxygen supply through reduced intestinal blood flow or oxygen short circuiting in the villus countercurrent exchange. Additionally, ischaemia may cause reperfusion injury, which can also damage the intestinal mucosa.

After hypovolaemia, or reduced cardiac output for other reasons (such as cardiac tamponade), the splanchnic blood supply might be insufficient to meet the tissues needs. It has been shown that the small intestinal blood supply has to be reduced by more than $50 \%$ to induce detectable tissue injury. ${ }^{6}$ If the blood flow reduction is less than $50 \%$, compensatory mechanisms will be enough to avert tissue injury. These mechanisms include increased oxygen extraction and oxygen redistribution within the intestinal wall to those areas with a high metabolic demand.

In haemorrhage or cardiac tamponade, or both the reduced delivery to the intestine may create sufficient hypoxia in the superficial part of the gut mucosa to cause tissue injury. ${ }^{7}$ Where ischaemia, however, was induced regionally in the small intestine by lowering the arterial inflow pressure, a very significant redistribution of intramural blood flow provides the mucosa with more oxygen. ${ }^{8}$ Moreover, in sepsis, blood flow to the superficial part of the mucosa has also been shown to be maintained well in animals developing intestinal mucosal injury. ${ }^{9}$ Furthermore, in regional intestinal ischaemia and in septic shock, the intestinal mucosal injury could be prevented by supplying the gut mucosa with small amounts of extra oxygen by intraluminal perfusion with oxygenated saline. Perfusion with nitrogen saturated saline had no such effect. ${ }^{910}$

The unusual situation where the blood supply to the superficial part of the mucosa is fairly well maintained but hypoxic injury still develops in this area within 1-2 hours of shock, may be explained by the peculiar vascular anatomy of the intestinal mucosa. The villus is supplied with a centrally located arterial vessel, which does not arborise until it reaches the tip of the villus. The blood flow is then drained through a subepithelial network of capillaries and venulas. The distance between these two sets of vessels with mainly opposite flow directions is less than $20 \mu$, which will permit diffusion equilibrium to take place for easily diffusable substances such as oxygen. A limiting factor is the time allowed to obtain diffusion equilibrium. In shock, the blood flow velocity is significantly reduced, ${ }^{11}$ and the prerequisite for a very effective short circuiting of oxygen at the base of the villi is present. A certain degree of short circuiting of oxygen in the villus countercurrent exchanger has been shown in the resting intestine, ${ }^{12}$ and a very low resting $\mathrm{pO}_{2}$ has been recorded at the tip of the villus. ${ }^{13}$ Thus, an increased effectiveness of the countercurrent exchanger during hypotensive states might well explain an almost anoxic environment at the tip of the villi despite a rather well maintained volume of blood flow to this region.

During recent years an additional mechanism causing impaired tissue oxygenation to the gut during sepsis has been described. Dahn et al ${ }^{14}$ provided strong indications for an increased oxygen demand of the splanchnic organs during sepsis compared with trauma not complicated by sepsis. Arvidsson et al ${ }^{15}$ could measure oxygen consumption in the gastrointestinal tract and in the liver during sepsis and haemorrhage. It could be shown that during sepsis induced by faecal peritonitis in pigs, the splanchnic oxygen consumption almost doubled within a couple of hours. For the intestine, the oxygen delivery was judged to be sufficient to meet the total oxygen demands of the tissue, under these experimental conditions. In sepsis, however, the hepatic needs for oxygen exceeded the delivery, and flow dependent hypoxia was expressed as increased lactate production. ${ }^{15}$ Recently, it was shown, using the tonometric technique for monitoring intramucosal $\mathrm{pH}$ (see later) that mucosal ischaemia occurred rapidly after sepsis despite maintained or increased oxygen delivery and increased oxygen consumption of the gut. ${ }^{16}$

The cause of the increased oxygen demand in the splanchnic area in sepsis is not fully understood. From a theoretical point of view, activated resident macrophages and invading white blood cells might account for at least part of the increased demand.

To summarise, in the critically ill, tissue N oxygenation of the splanchnic organs is threatened by several simultaneous mechanisms 0 causing intestinal ischaemia. Blood flow delivery to the area might be impaired. The mucosal countercurrent exchanger causes a further decrease of oxygen delivery to the superficial part of the mucosa. In the septic state there is, in addition, an increased demand for oxygen in the splanchnic area. Moreover, sepsis causes decreased extraction and utilisation of oxygen by the tissue.

\section{Reperfusion injury}

During the past 10 years, there has been increasing evidence that, in addition to the injury taking place during ischaemia, intestinal injury also takes place at reperfusion. ${ }^{17} 18$ The 


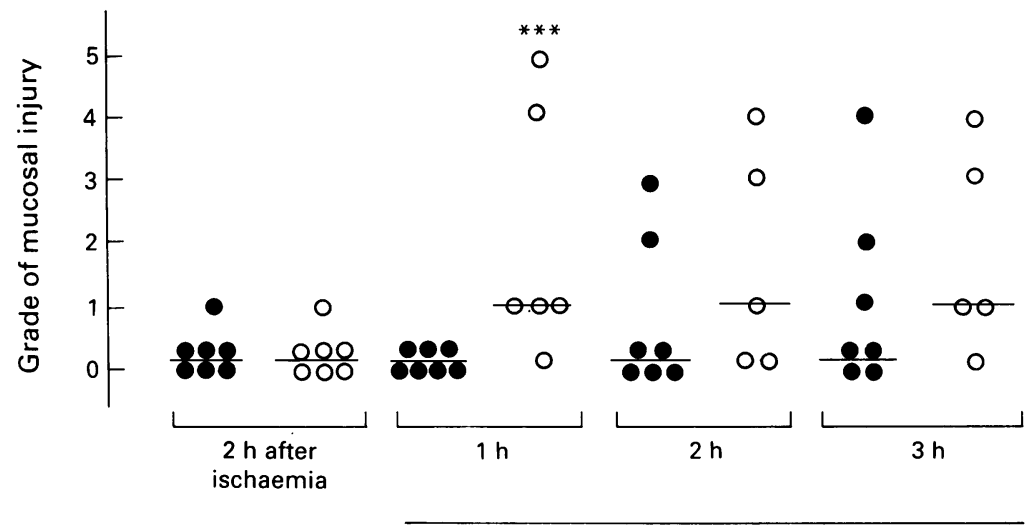

Reperfusion

Figure 3: Effect of chronic pancreatic duct ligation (filled circles) on intestinal mucosal injury developed at haemorrhagic hypotension followed by reperfusion in pigs. $\star \star \star$ denotes $p>0.01$ in comparison between the groups. Open circles denote control animals subjected to a previous sham operation and haemorrhagic hypotension of similar extent and duration as the pancreatic duct ligated animals.

mechanism underlying the reperfusion component of the total tissue injury ${ }^{3}$ is generally believed to be increased generation of oxygen derived free radicals. Probably the most important mechanism for increased generation of oxygen radicals in the intestine is the hypoxanthine-xanthine oxidase system. ${ }^{17}$

The reperfusion component of ischaemic injury is more pronounced after partial than total intestinal ischaemia. ${ }^{319}$ Based on available experimental data, it is reasonable to conclude that only the superficial mucosa is subjected to detectable reperfusion injury. If the ischaemic injury is severe enough to cause injury to deeper layers of the gut wall than the villi, further injury at reperfusion is probably not detectable. Similarly, if the ischaemic injury by itself does not cause any detectable injury, probably such injury will not be found after reperfusion. As reviewed elsewhere ${ }^{20}$ such a 'window of reperfusion injury' has also been shown for other organs.

\section{Effect of intraluminal proteases}

Bounous et al showed that intraluminal proteases, especially trypsin, were important for the development of intestinal mucosal injury during shock. They showed that aprotinin, a proteolytic enzyme inhibitor, placed in the lumen before induction of shock, as well as pancreatic duct ligation one week before intestinal ischaemia, could prevent the appearance of intestinal mucosal injury (see ref 21 ). At that time, however, the role of the ischaemia $v$ the reperfusion phase of the total ischaemic tissue injury was not considered. Later, it was suggested that the proteolytic activity in the lumen might be especially important for the rapid conversion of xanthine dehydrogenase to xanthine oxidase (D to $O$ conversion) and, consequently, intraluminal proteases would be of significant importance for the reperfusion phase of a total ischaemic injury. ${ }^{22}$ Recently, this hypothesis was tested, and it was shown that, after two hours of haemorrhage hypotension, there was no difference between animals with a chronic pancreatic duct ligation and those without. Those with an intact pancreatic duct, however, did develop reperfusion injury more rapidly than those with a chronic duct ligation (Fig 3). The second group of animals did finally (after three hours) develop an exacerbated tissue injury during the reperfusion phase. ${ }^{23}$ Absence of intraluminal pancreatic proteases in the gut lumen thus delayed but did not avert the development of reperfusion injury.

\section{Monitoring of gut ischaemia}

These considerations strongly suggest that, especially in sepsis, a knowledge of blood flow does not necessarily provide any information about the ischaemic state of the intestine. A technique permitting the detection and monitoring of intestinal ischaemia has recently been described by Fiddian-Green. ${ }^{24}$ This technique is reasonably non-invasive and is easily used in the clinical setting. Validation has been described by Antonsson et al. ${ }^{25}$ The tonometer has a carbon dioxide permeable silicon balloon on its tip. Combined with a nasogastric tube, it can easily be placed in the stomach. The silicon balloon is connected with the outside through an impermeable tube. If the balloon is filled with saline, carbon dioxide equilibrates rapidly over the silicone membrane of the balloon between the saline content and the lumen. The lumenal $\mathrm{pCO}_{2}$ is in turn, in equilibrium with $\mathrm{pCO}_{2}$ of the superficial mucosa. By measuring $\mathrm{pCO}_{2}$ in the balloon and arterial bicarbonate concentration, the $\mathrm{pH}$ can be calculated with the HendersonHasselbalch equation.

\section{Effects of enteral nutrition during ischaemia}

There is no randomised trial illustrating a clear cut benefit to the ischaemic intestine by enteral feeding. There are several indirect indications, however, that early enteral feeding might be important in limiting the sequelae of intestinal ischaemic injury. It has been noticed that parenteral nutrition in itself might cause mucosal tissue injury closely similar to that found in ischaemia. ${ }^{26}$ This injury could be attenuated by enteral feeding. ${ }^{26}$ Similarly, it has been shown that total parenteral nutrition promotes bacterial translocation from the gut. ${ }^{27}$ Furthermore, it has been shown that enteral nutrition reduces septic morbidity after major abdominal trauma compared with total parenteral nutrition. ${ }^{28}$ Of special interest are the reports of the importance of the amino acid glutamine on the gut mucosal integrity. Supplying this amino acid in parenteral nutrition has been reported to attenuate mucosal ischaemic injury. ${ }^{29}$ There is indirect support for the concept that glutamine supplementation in diets used for enteral nutrition has a similarly beneficial effect. ${ }^{30}$

Figure 3 is reproduced from Montgomery, Borgström and Haglund. Gastroenterology 1992; 102: 216-22 (ref 23), with permission.

Wells CL, Maddaus MA, Simmons RL. Bacterial translocation. In: Marston A, Bulkley GB, Fiddian-Green RG, Haglund $U$, eds Splanchnic ischemia and multiple organ failure London: Edward Arnold, 1989: 195-204. 
2 Haglund U. Gastro-intestinal mucosal injury in shock. In: Vincent $\mathrm{J}$, ed. Update in intensive care and emergency med icine. Berlin, Heidelberg: Springer-Verlag, 1991: 154-60.

3 Haglund U, Bulkley GB, Granger DN. On the pathophysiology of intestinal ischemic injury. Acta Chir Scand 1987; 153: $321-4$.

4 Chiu CJ, McArdle AH, Brown R, Scott HJ, Gurd FN. Intestinal mucosal lesion in low-flow states. Arch Surg 1970; 101: 478-83.

5 Park PO, Haglund U. Regeneration of small bowel mucosa after intestinal ischemia. Crit Care Med 1992; 20: 135-9.

6 Bulkley GB, Kvietys PR, Parks DA, Perry MA, Granger DN. Relationship of blood flow and oxygen consumption to ischemic injury in the canine small intestine. Gastroenterology 1985; 89: 852-7.

7 Bailey RW, Bulkley GB, Hamilton SR, Morris JB, Haglund UH. Protection of the small intestine from nonocclusive mesenteric ischemic injury due to cardiogenic shock. $\mathrm{Am}$ mesenteric ischemic injury

8 Redfors S, Hallbäck DA, Haglund U, Jodal M, Lundgren $\mathrm{O}$. Blood flow distribution, villous osmolality and fluid and electrolyte transport in the cat small intestine during regional hypotension. Acta Physiol Scand 1984; 121: 193-209.

9 Falk A, Redfors S, Myrvold HE, Haglund U. Small intestinal mucosal lesions in feline septic shock; a study on the pathogenesis. Circ Shock 1985; 17: 327-37.

10 Haglund U, Abe T, Åhrénc C, et al. The intestinal mucosal lesions in shock: I. Studies on the pathogenesis. Eur Surg Res $1984 ; 8: 435-47$.

11 Haglund U, Jodal $M$, Lundgren $O$. The small bowel in arterial hypotension and shock. In: Shephaerd AP, Granger DN, eds. Physiology of the intestinal circulation. New York: Raven Press, 1984: 305-19.

12 Kampp M, Lundgren O, Nilsson NJ. Extravascular shunting of oxygen in the small intestine of the cat. Acta Physiol Scand 1968; 72: 396-403.

13 Bohlen HG. Intestinal tissue $\mathrm{pO}_{2}$ and microvascular responses during glucose exposure. Am f Physiol 1980 238: $\mathrm{H} 164-71$.

14 Dahn MS, Lange MP, Lodell K, Hans B, Jacobs LA, Mitchell RA. Splanchnic and total body oxygen consumption differences in septic and injured patients. Surgery tion differences in

15 Arvidsson D, Rasmussen I, Almqvist P, Niklasson F, Haglund U. Splanchnic oxygen consumption in septic and hemorrhagic shock. Surgery 1991; 2: 190-7.

16 Rasmussen I, Haglund U. Early gut ischemia in experimental fecal peritonitis. Circ Shock 1992; 38: 22-8.
17 Granger DN, Rutili G, McCord JM. Superoxide radicals in feline intestinal ischemia. Gastroenterology 1981; 81: 22-9.

18 Schoenberg MH, Fredholm B, Haglund U, et al. Studies on the oxygen radical mechanism involved in the small intestinal reperfusion damage. Acta Physiol Scand 1985, 124: 581-9.

19 Park PO, Haglund U, Bulkley GB, Fält K. The sequence of development of intestinal tissue injury following strangulation ischemia and reperfusion. Surgery 1990; 107: 574-80.

20 Haglund U, Gerdin B. Oxygen-free radicals (OFR) and circulatory shock. Circ Shock 1991; 34: 405-11.

21 Bounous G. Acute necrosis of the intestinal mucosa. Gastroenterology 1982; 82: 1457-67.

22 Parks DA, Williams TK, Beckman JS. Conversion of xanthine dehydrogenase to oxidase in ischemic rat intestine: a reevaluation. $A m$ f Physiol 1988; 254: G768-74.

23 Montgomery A, Borgström A, Haglund U. Pancreatic proteases and intestinal mucosal injury after ischemia and reperfusion in the pig. Gastroenterology 1992; 102: 216-22.

24 Fiddian-Green RG. Studies in splanchnic ischemia and multiple organ failure. In: Marston A, Bulkley GB, Fiddian-Green RG, Haglund U, eds. Splanchnic ischemia and multiple organ failure. London: Edward Arnold, 1989: 350-63.

25 Antonson JB, Boyle CC, Kruithoff KL, Wang H, Sacristan E, Rothshild HR, et al. Validation of tonometric measurement of gut intramural $\mathrm{pH}$ during endotoxemia and mesenteric occlusion in the pig. Am $\mathcal{F}$ Physiol 1990; 259: G519-23.

26 Nobumichi $\mathrm{H}$, Masaharu N, Manabu N, et al. Structural and functional alterations in the gut of parenterally or enterally fed rats. $\mathcal{F}$ Surg Res $1989 ; 47: 129-33$.

27 Alverdy JC, Aoys E, Moss GS. Total parenteral nutrition promotes bacterial translocation from the gut. Surgery 1988; 104: 185-90.

28 Moore FA, Moore EE, Jones TN, et al. TEN versus TPN following major abdominal trauma - reduces septic morbidity. F Trauma 1989; 29: 916-23.

29 Hwang TL, O'Dwyer ST, Smith RJ, et al. Preservation of the small bowel mucosa using glutamine-enriched parenteral nutrition Surg Forum 1986; 37: 56-8.

30 Rombeau JL. A review of the effects of glutamine-enriched diets on experimentally induced enterocolitis. FPEN 1990; 14: 100S-5S.

\section{(n)}

